\title{
Pharmacokinetic/pharmacodynamic analysis of adjuvant pegylated interferon $\alpha-2 b$ in patients with resected high-risk melanoma
}

\author{
A. I. Daud · C. Xu $\cdot$ W.-J. Hwu $\cdot$ P. Urbas $\cdot$ S. Andrews $\cdot$ \\ N. E. Papadopoulos $\cdot$ L. C. Floren $\cdot$ A. Yver $\cdot$ \\ R. C. DeConti $\cdot$ V. K. Sondak
}

Received: 7 January 2010 / Accepted: 1 April 2010 / Published online: 28 May 2010

(c) The Author(s) 2010. This article is published with open access at Springerlink.com

\begin{abstract}
Purpose High-dose pegylated interferon $\alpha-2 \mathrm{~b}$ (peginterferon $\alpha-2 b)$ significantly decreased disease recurrence in patients with resected stage III melanoma in a clinical study. We investigated the pharmacokinetics (PK) and safety of high-dose peginterferon $\alpha-2 b$ in patients with high-risk melanoma.

Methods For PK analysis, 32 patients received peginterferon $\alpha-2 \mathrm{~b} \quad 6 \mu \mathrm{g} /(\mathrm{kg}$ week) subcutaneously for 8 weeks (induction) then $3 \mu \mathrm{g} /(\mathrm{kg}$ week) for 4 weeks (maintenance). PK profiles were determined at weeks 1, 8, and 12. Exposure-response relationships between peginterferon $\alpha-2 b$ and absolute neutrophil count (ANC) and alanine aminotransferase (ALT) level were also studied.
\end{abstract}

Electronic supplementary material The online version of this article (doi:10.1007/s00280-010-1326-9) contains supplementary material, which is available to authorized users.

\section{A. I. Daud $(\bowtie)$}

Division of Hematology/Oncology,

University of California, San Francisco, San Francisco, CA, USA

e-mail: adaud@medicine.ucsf.edu

C. Xu $\cdot$ A. Yver

Merck Research Laboratories, Kenilworth, NJ, USA

W.-J. Hwu · N. E. Papadopoulos

M.D. Anderson Cancer Center, Houston, TX, USA

P. Urbas · S. Andrews · R. C. DeConti · V. K. Sondak

Department of Cutaneous Oncology,

H. Lee Moffitt Cancer Center and Research Institute,

Tampa, FL, USA

P. Urbas · S. Andrews · R. C. DeConti · V. K. Sondak

Department of Oncologic Sciences,

University of South Florida College of Medicine,

Tampa, FL, USA
Results Peginterferon $\alpha-2 \mathrm{~b}$ was well-absorbed following SC administration, with a median $T_{\max }$ of $24 \mathrm{~h}$. Mean half-life estimates ranged from 43 to $51 \mathrm{~h}$. The accumulation factor was 1.69 after induction therapy. PK parameters showed moderate interpatient variability. PK profiles were described by a one-compartmental model with firstorder absorption and first-order elimination. Toxicity was profiled and was acceptable; observed side effects were similar to those previously described. Dose reduction produced proportional decreases in exposure and predictable effects on ANC in an Imax model; however, a PK/ pharmacodynamic (PK/PD) relationship between peginterferon $\alpha-2 b$ and ALT could not be established with high precision.

\author{
L. C. Floren \\ Department of Bioengineering and Therapeutic Sciences, \\ University of California, San Francisco, San Francisco, CA, USA \\ Present Address: \\ A. Yver \\ AstraZeneca Pharmaceuticals LP, Wilmington, DE, USA \\ A. I. Daud \\ Division of Hematology/Oncology, Melanoma Program, \\ University of California, San Francisco, MTZ A741, \\ Box 1770, 1600 Divisadero St, San Francisco, CA 94143, USA
}


Conclusions Peginterferon $\alpha-2 b$ was well-absorbed and sustained exposure to peginterferon $\alpha-2 b$ was achieved with the doses tested. These data confirm and extend previous PK observations of peginterferon $\alpha-2 b$ in melanoma and solid tumors. Our PK/PD model of exposure and ANC effect provides useful information for prediction of peginterferon $\alpha$-2b-related hematologic toxicity.

Keywords Peginterferon $\alpha$-2b · Melanoma .

Adjuvant therapy $\cdot$ Pharmacokinetics $\cdot$ Pharmacodynamics

\section{Introduction}

The conjugation of polyethylene glycol (PEG) to therapeutically useful proteins has been widely employed to reduce clearance and increase systemic exposure while preserving biologic activity [1]. The addition of a $12 \mathrm{kDa}$ mono-methoxy-PEG linker to interferon produces a predominantly mono-pegylated prodrug molecule that retains the activity of the parent interferon and has a prolonged half-life that lends itself to weekly administration [2].

High-dose recombinant (native) interferon $\alpha-2 b$ is widely used and is approved by the US Food and Drug Administration as adjuvant therapy in patients with highrisk resected melanoma, based on its effect on disease-free survival (DFS) and overall survival (OS) in the pivotal Eastern Cooperative Oncology Group (ECOG) 1684 trial [3]. Further trials established the effect of interferon $\alpha-2 b$ on DFS, although its effects on OS are somewhat controversial, with only modest improvements in OS versus observation [3-7]. High-dose interferon $\alpha-2 b$ regimens are associated with high toxicity [8] and a cumbersome, threetimes-weekly maintenance dosing schedule. Additionally, several studies have demonstrated that peginterferon $\alpha-2 b$ is more effective than nonpegylated interferon in treating hepatitis [9-12] owing to the altered pharmacokinetic (PK) profile and resultant prolonged drug exposure [11-13]. Peginterferon $\alpha$-2b has also shown promise in the treatment of various solid tumors [2, 13, 14]. Given this information, investigators started studying high-dose peginterferon $\alpha-2 b$ for treatment of melanoma in the late 1990s [2, 13, 15].

The recently reported European Organisation for Research and Treatment of Cancer (EORTC) 18991 phase III trial studied the efficacy of peginterferon $\alpha-2 b$ in highrisk melanoma. Improvement in recurrence-free survival (RFS) was seen in patients with node-positive melanoma treated for up to 5 years with high-dose peginterferon $\alpha-2 b$ compared with observation alone [15]. Therefore, highdose peginterferon $\alpha$-2b [6 $\mu \mathrm{g} /(\mathrm{kg}$ week) for 8 weeks followed by $3 \mu \mathrm{g} /(\mathrm{kg}$ week) for up to 5 years] could become a new option for the adjuvant treatment of stage III patients with high-risk melanoma. The dose used in the EORTC
18991 trial was substantially higher than that generally used for the treatment of hepatitis $\mathrm{C}[1.5 \mu \mathrm{g} /(\mathrm{kg}$ week $)]$; $6 \mu \mathrm{g} /(\mathrm{kg}$ week) subcutaneous (SC) dosing is expected to provide an exposure (area under the curve; AUC) similar to high-dose native interferon $\alpha$-2b $180 \mathrm{MIU} /$ week intravenously, which is the weekly dose a patient with a body surface area of $1.8 \mathrm{~m}^{2}$ would receive in the first 4 weeks of standard high-dose adjuvant therapy for melanoma [20 MIU/(m² day) five times weekly] [13]. A recent publication reported limited PK data from a small subset of patients $(7 \%)$ receiving peginterferon $\alpha$-2b during the maintenance phase in the 18991 study [16]. The results indicated sustained exposure to peginterferon $\alpha$-2b during long-term adjuvant treatment, with dose-related changes in serum trough concentrations. Mean serum concentrations were similar to those observed in patients with hepatitis $C$; high inter-subject variability was observed, consistent with hepatitis C studies. However, PK analysis was not planned in the 18991 study protocol and was assessed using serum samples obtained during routine clinical visits 1-6 months apart for exploratory analysis of prognostic factors; therefore multiple sequential samples were not available for all patients, and blood sampling was not always well-timed relative to administration of the weekly drug dose. These factors have important implications for the trough level estimates in the $18991 \mathrm{PK}$ analysis.

To provide data on the actual exposure and $\mathrm{PK}$ parameter estimates for patients treated with this high-dose schedule of peginterferon $\alpha-2 b$, we conducted a prospective, single-arm, open-label trial in resected, high-risk, stages II and III melanoma. Given that absolute neutrophil count (ANC) reduction and alanine aminotransferase (ALT) elevations are two of the most common and significant dose-limiting organ toxicities with interferon treatment, and possibly peginterferon $\alpha-2 b$ [17], we explored PK/pharmacodynamic (PK/PD) models of ANC and ALT changes following peginterferon $\alpha-2 b$ SC administration and attempted to model the expected effect of dose modification on bone marrow suppression and hepatic toxicity.

\section{Methods}

Trial design

This prospective, open-label, single-arm, phase II PK trial was conducted at four sites worldwide. The primary goal was to determine the PK profile of peginterferon $\alpha-2 b$ when administered at the EORTC 18991 trial dose and schedule in patients with high-risk melanoma. The secondary objective was to assess the safety of this regimen. Additionally, exploratory modeling of the $\mathrm{PD}$ relationship between peginterferon $\alpha-2 b$ exposure and ANC and ALT was carried out. 
The trial was approved by the local institutional review boards and registered at http://www.clinicaltrials.gov (NCT00457418). The trial opened in February 2007 and completed accrual in September 2007.

\section{Patients}

Eligible patients were $\geq 18$ years old and had histologically documented American Joint Committee on Cancer stage IIB, IIC, IIIA, IIIB, or IIIC melanoma, an ECOG performance status of 0 or 1 , and adequate bone marrow, renal, and hepatic function. Full lymphadenectomy had to be performed within 90 days prior to starting therapy. Patients with a history of treatment with any interferon, chemotherapy, or immunotherapy, and those with melanoma that could not be completely surgically resected were excluded, as were those with preexisting autoimmune disease or psychiatric conditions. This study was conducted in accordance with Good Clinical Practice guidelines and signed informed consent was obtained prior to treatment initiation.

\section{Treatment}

Patients received peginterferon $\alpha-2 \mathrm{~b} 6 \mu \mathrm{g} / \mathrm{kg}$ SC once weekly during an 8-week induction period, followed by a maintenance dosage of $3 \mu \mathrm{g} / \mathrm{kg}$ SC once weekly for the remainder of the treatment period for a maximum of 5 years. The first 12 weeks constituted the PK phase ( 8 weeks of induction and 4 weeks of maintenance); the remainder was the post-PK phase. Dose modification guidelines were provided to manage toxicities, allowing patients to continue treatment while maintaining an ECOG performance status score of 0 or 1, per the EORTC 18991 trial [15]. Treatment was withheld if hematologic toxicity occurred: white blood cells $<1 \times 10^{9} / \mathrm{L}$; ANC $<0.5 \times 10^{9} /$ $\mathrm{L}$; platelets $<50 \times 10^{9} / \mathrm{L}$. After recovery, treatment could be restarted one dose level down; subsequent dose escalations were not permitted after dose reduction for hematologic toxicity (see online resource material-supplementary methods).

Safety evaluation

The cutoff date for safety data presented here was May 2008 . Baseline evaluations included history, physical examination, complete blood count with differential, lipid profiles, and renal, hepatic, and thyroid function assessments (see online resource material—supplementary methods).

Blood collection and analysis

Blood samples for PK analysis were collected predose and at $24,48,96$, and $168 \mathrm{~h}$ postdose during weeks 1,8 , and 12 .
In addition, a predose sample was drawn at weeks 11 and 12 to determine trough levels (see online resource material-supplementary methods).

Noncompartmental PK analysis

PK parameters for individual serum peginterferon $\alpha-2 b$ concentration-time data were estimated using noncompartmental (NCA) methods (WinNonlin software, version 4, Pharsight, Mountain View, CA). Samples with concentrations below the limit of quantitation of the bioanalytical assay were assigned a concentration of $0 \mathrm{pg} / \mathrm{mL}$. The following parameters were calculated from the concentrationtime data using this NCA: area under the curve during the dosing interval $\left(\mathrm{AUC}_{\mathrm{tau}}\right)$, maximal serum concentration $\left(C_{\text {max }}\right)$, average concentration within dosing interval $\left(C_{\text {avg }}\right)$, minimum (trough) serum concentration $\left(C_{\min }\right)$, observed time to achieve maximal serum concentration $\left(T_{\max }\right)$, mean apparent clearance $(\mathrm{CL} / \mathrm{F})$, terminal half-life $\left(t_{1 / 2}\right)$, and accumulation factor $(R)$ [18]. For weeks $1-8$ (induction) $R$ was defined as the ratio of the $\mathrm{AUC}_{0-168 \mathrm{~h}}$ for week 8 divided by the $\mathrm{AUC}_{0-168 \mathrm{~h}}$ for week 1 . At week 1, the single dose $\mathrm{CL} / \mathrm{F}$ was calculated using dose/AUC $\infty$. At weeks 8 and $12, \mathrm{CL} / \mathrm{F}$ was calculated as dose/AUC ${ }_{0-168 \mathrm{~h}}$. CL/F was not determined for those patients whose elimination $t_{1 / 2}$ could not be accurately determined at week 1 .

\section{Compartmental PK analysis}

A one-compartment model and a two-compartment model were evaluated using WinNonlin software. A one-compartment model with first-order absorption and first-order elimination (no lag time) appropriately described the PK profiles for peginterferon $\alpha-2 b$ following multiple SC dosing (see online resource material—supplementary methods and supplementary Table 1). Individual serum concentrations at weeks 1,8 , and 12 and trough concentration at weeks 10 and 11 were used to obtain the individual fitted PK parameters (data not shown). Once the parameter values were determined, the predicted serum concentration-time profiles were generated and compared with actual data obtained to assess the usefulness of the PK model. The initial PK parameter estimates were based on either the relevant literature or the initial value calculated by the WinNonlin software.

\section{PD modeling strategy and PK/PD analysis}

Blood samples were collected at baseline and approximately every week subsequently to determine ANC and ALT levels. PK/PD models were developed in order to characterize the time course of ANC or ALT response after $\mathrm{SC}$ administration of peginterferon $\alpha-2 b$, using WinNonlin 
software. For both ANC and ALT, model selection was driven by the available PK and PD data and was based on: (1) graphical goodness-of-fit analysis, (2) estimated uncertainty in parameter estimates as reported by $\% \mathrm{CV}$, (3) Akaike information criteria (AIC), and (4) plausibility of parameter estimates. Initial parameter estimates were based on the relevant literature or initial values provided by the WinNonlin software and their plausibility ascertained. Since only two dose levels were specified by this study protocol (excluding dose reductions), we pooled data from all 32 patients in our study with those of a different trial [2], which used a wide dose range in patients with solid tumors, to better describe the dose-response relationship. Previously published steady-state exposure values $\left(\mathrm{AUC}_{\text {tau }}\right.$ at week 4) from 34 patients with solid tumors treated with doses ranging from 0.75 to $7.5 \mu \mathrm{g} /(\mathrm{kg}$ week) (3-12 patients in each dose group) [2] were used, along with the corresponding ANC data from the same patients [unpublished data, provided by Schering-Plough (now Merck \& Co.)]. In this previously published study, blood samples were collected predose (hour 0 ) and at $24,48,72$, and $168 \mathrm{~h}$ postdose during weeks 1 and 4 . Six of the 34 patients with solid tumors were melanoma patients.

\section{Effects of peginterferon $\alpha-2 b$ exposure on ANC}

The exposure-response relationship between steady-state exposure to peginterferon $\alpha-2 b\left(\mathrm{AUC}_{\mathrm{tau}}\right)$ and changes in ANC from baseline was assessed at week 8 [dose $6 \mu \mathrm{g} /$ ( $\mathrm{kg}$ week)] and week 12 [3 $\mu \mathrm{g} /(\mathrm{kg}$ week)]. The inhibitory Imax model was used to describe the exposure-response relationship of ANC change as \% of baseline and peginterferon $\alpha-2 \mathrm{~b} \mathrm{AUC}_{\text {tau }}$ (see online resource material-supplementary methods and supplementary Table 1 ).

Based on serum concentrations and ANC data obtained from patients in this study, a fully integrated PK/PD model was used to describe the effect of peginterferon $\alpha-2 b$ on ANC over time. The PK component was a one-compartment model with first-order absorption and first-order elimination. The PD component of the model is an indirect inhibition response model driven by the PK profiles $[19,20]$.

\section{Effects of peginterferon $\alpha-2 b$ exposure on $A L T$}

Again, a fully integrated PK/PD model was used to describe the effect of peginterferon $\alpha-2 b$ on the time course of ALT change. The PK and PD components for the model were as described for ANC above.

\section{Statistical analyses}

The protocol-defined target sample size was 12 subjects; assuming an inter-subject coefficient of variation of $40 \%$, this was deemed sufficient to define the PK parameters such that the $90 \%$ CI would be within $24 \%$ of the mean. Therefore, between 20 and 30 subjects were to be enrolled to ensure that 12 subjects would receive the full scheduled dose of peginterferon $\alpha-2 b$ for 12 weeks. Summary statistics are provided for PK parameters by dose and week with the mean and \% CV for the serum concentration data at each sampling time. Steady state was assessed using log-transformed $C_{\min }$ for weeks 10, 11, and 12 using an ANOVA model extracting effects due to week and patient. Ratio estimates and corresponding 90\% CI were provided for each week versus the average of the subsequent weeks. ANC and ALT data were summarized by week.

\section{Results}

Patient characteristics and treatment exposure

Thirty-two patients participated in this trial. Baseline patient demographics are summarized in Table 1. Dose intensity was calculated by dividing the total dose received in each phase by the corresponding time period for each individual subject. Median dose intensity was $5.8 \mu \mathrm{g} /$ ( $\mathrm{kg}$ week) during the induction phase (weeks 1-8), $3.0 \mu \mathrm{g} /$ (kg week) during the PK maintenance phase (weeks 9-12), and $2.9 \mu \mathrm{g} /(\mathrm{kg}$ week) during the post-PK phase. Most patients $(84 \%)$ received $7-8$ weeks of induction therapy. The median duration of treatment was 8.0 weeks (range 5.0-8.4) for induction and 4.0 weeks (range 1.0-4.1) for the PK maintenance phase.

A total of 30 patients participated in the post-PK phase (two patients did not enter follow-up), of whom 15 (50\%) discontinued therapy: $5(17 \%)$ each due to adverse events (AEs), disease progression, and patient preference. By July 2008 (last database lock), 15 (50\%) patients remained on treatment at a median of 13 months after beginning therapy.

Safety

Safety analyses were performed on all 32 patients. The observed side effects of peginterferon $\alpha-2 b$ were similar to those previously described [2, 13, 15]. All patients reported $\geq 1$ treatment-emergent AEs (TEAEs) of any grade; $22(69 \%)$ reported grade 3 or 4 TEAEs. TEAEs reported in $\geq 10 \%$ of patients are presented in Table 2 . The most commonly occurring AEs included fatigue, fever, chills, headache, anorexia, and nausea. Decreases in ANC (all grades) were observed in seven (22\%) patients; decreases of grades 3 and 4 were observed in three $(9 \%)$ patients.

Serious AEs were reported in six (19\%) patients: lower limb and wrist fracture (one patient); drug hypersensitivity, 
Table 1 Baseline demographics and tumor stage

\begin{tabular}{ll}
\hline Characteristic & $n=32$ \\
\hline Age (years) & \\
Mean \pm SD & $49 \pm 13$ \\
Median (range) & $50(20-77)$ \\
18-65 years, $n(\%)$ & $29(91)$ \\
$\geq 65$ years, $n(\%)$ & $3(9)$ \\
Weight $(\mathrm{kg})$ & \\
Mean \pm SD & $84 \pm 21$ \\
Median (range) & $82(46-137)$ \\
Missing & 1 \\
Sex, $n(\%)$ & \\
Female & $10(31)$ \\
Male & $22(69)$ \\
Race, $n(\%)$ & \\
White & $31(97)$ \\
Black or African American & $1(3)$ \\
Tumor stage, $n(\%)$ & \\
IIB & $2(6)$ \\
IIIA & $5(16)$ \\
IIIB & $19(59)$ \\
IIIC & $6(19)$ \\
\hline
\end{tabular}

bronchitis, and dehydration (one patient); urticaria (one patient); cholecystitis (one patient); and atrial fibrillation (two patients). All serious AEs were grade 3 with the exception of one grade 2 atrial fibrillation.

AEs leading to dose modification were reported in 17 (53\%) patients and included fatigue and increased ALT level (four patients (13\%) each). Decreases in ANC (all grades) led to dose reduction in two (6\%) patients. AEs leading to treatment discontinuation were reported in seven (22\%) patients and included lower leg fracture, atrial fibrillation, fatigue, supraventricular tachycardia, blurred vision, staphylococcal infection, and hypertriglyceridemia (one patient each). No treatment-related deaths were reported.

\section{Pharmacokinetics}

Of the 32 patients enrolled on the study, five (16\%) patients discontinued treatment during the initial 12 weeks (PK phase): two $(6 \%)$ due to AEs, one $(3 \%)$ due to disease progression, and two $(6 \%)$ due to noncompliance with protocol. Another seven (22\%) had missed/reduced doses, or missed one or more specified PK sampling points, during the PK phase. Data from the remaining 20 patients who received full doses and provided all blood samples in the PK phase were used for the primary PK analyses, as per the protocol (a minimum of 12 patients was required). Data
Table 2 Treatment-emergent adverse events reported in $\geq 10 \%$ of patients in the total study population $(n=32)$

\begin{tabular}{|c|c|c|}
\hline $\begin{array}{l}\text { Treatment-emergent } \\
\text { adverse events }\end{array}$ & $\begin{array}{l}\text { All grades } \\
n(\%)\end{array}$ & $\begin{array}{l}\text { Grade } 3 / 4 \\
n(\%)\end{array}$ \\
\hline \multicolumn{3}{|l|}{ Hematologic } \\
\hline Neutropenia & $7(22)$ & 2/1 (9) \\
\hline Leukopenia $^{\mathrm{a}}$ & $3(9)$ & $3 / 0(9)$ \\
\hline \multicolumn{3}{|l|}{ Gastrointestinal } \\
\hline Anorexia & $28(88)$ & 0 \\
\hline Constipation & $4(13)$ & 0 \\
\hline Diarrhea & $19(59)$ & 0 \\
\hline Nausea & $22(69)$ & 0 \\
\hline Vomiting & $6(19)$ & 0 \\
\hline Dehydration & $4(13)$ & $1 / 0(3)$ \\
\hline \multicolumn{3}{|l|}{ Constitutional symptoms } \\
\hline Insomnia & $9(28)$ & 0 \\
\hline Chills & $26(81)$ & 0 \\
\hline Fatigue & $29(91)$ & $3 / 0(9)$ \\
\hline Influenza-like illness & $6(19)$ & 0 \\
\hline Night sweats & $4(13)$ & 0 \\
\hline Fever & $27(84)$ & 0 \\
\hline \multicolumn{3}{|l|}{ Metabolic/laboratory } \\
\hline Alanine aminotransferase & $9(28)$ & 4/0 (13) \\
\hline Aspartate aminotransferase & $6(19)$ & $2 / 0(6)$ \\
\hline Triglyceride elevations & $22(69)$ & $3 / 4(22)$ \\
\hline \multicolumn{3}{|l|}{ Pain } \\
\hline Headache & $23(72)$ & 0 \\
\hline Arthralgia & $5(16)$ & 0 \\
\hline Back & $7(22)$ & 0 \\
\hline Myalgia & $18(56)$ & 0 \\
\hline \multicolumn{3}{|l|}{ Neurology } \\
\hline Dizziness & $4(13)$ & 0 \\
\hline Anxiety & $7(22)$ & 0 \\
\hline Somnolence & $5(16)$ & 0 \\
\hline Depression & $11(34)$ & 0 \\
\hline \multicolumn{3}{|l|}{ Psychiatric } \\
\hline Insomnia & $9(28)$ & 0 \\
\hline \multicolumn{3}{|l|}{ Sexual/reproductive function } \\
\hline Erectile dysfunction & $4(13)$ & 0 \\
\hline \multicolumn{3}{|l|}{ Pulmonary/upper respiratory } \\
\hline Cough & $12(38)$ & 0 \\
\hline Dyspnea & $7(22)$ & 0 \\
\hline \multicolumn{3}{|l|}{ Skin/dermatology } \\
\hline Injection site reaction & $6(19)$ & 0 \\
\hline Pruritus & $7(22)$ & 0 \\
\hline Rash & $8(25)$ & $1 / 0(3)$ \\
\hline
\end{tabular}

$\bar{a}$ Leukopenia occurred at a frequency of $<10 \%$ but is included as a treatment-emergent adverse event of interest

from all 32 patients were pooled with data from patients with advanced solid tumors $(n=34)$ [2] to help construct the PK/PD model. 
Table 3 Pharmacokinetic parameters of peginterferon $\alpha$-2b following once-weekly subcutaneous dosing $(n=20)$

\begin{tabular}{|c|c|c|c|c|c|c|c|c|c|}
\hline \multirow[t]{2}{*}{ Week } & \multirow[t]{2}{*}{ Dose $(\mu \mathrm{g} / \mathrm{kg})$} & \multicolumn{8}{|c|}{ Arithmetic mean (\%CV) } \\
\hline & & $\mathrm{AUC}_{\text {tau }}(\mathrm{pg} \mathrm{h} / \mathrm{mL})$ & $C_{\max }(\mathrm{pg} / \mathrm{mL})$ & $C_{\text {avg }}(\mathrm{pg} / \mathrm{mL})$ & $C_{\min }(\mathrm{pg} / \mathrm{mL})$ & $T_{\max }(\mathrm{h})^{\mathrm{a}}$ & $\mathrm{CL} / \mathrm{F}(1 / \mathrm{h} \mathrm{kg})$ & $t_{1 / 2}(\mathrm{~h})$ & $R^{\mathrm{b}}$ \\
\hline 1 & 6 & $324,000(38)$ & $3,980(44)$ & $1,930(38)$ & NA & $24(24-72)$ & $0.0186(50)^{\mathrm{c}}$ & $46(24)^{\mathrm{c}}$ & NA \\
\hline 8 & 6 & $462,000(35)$ & $5,070(51)$ & $2,750(35)$ & $1,300(56)$ & $24(24-72)$ & $0.0152(48)$ & $51(18)^{d}$ & $1.69(64)$ \\
\hline 12 & 3 & $235,000(24)$ & $2,620(33)$ & $1,400(24)$ & $626(43)^{\mathrm{d}}$ & $24(24-48)$ & $0.0135(25)$ & $43(19)$ & NA \\
\hline
\end{tabular}

$\mathrm{AUC}_{\mathrm{tau}}$, area under the concentration-time curve during the dosing interval $(168 \mathrm{~h}) ; C_{\mathrm{avg}}$, average concentration within dosing interval; $C_{\mathrm{max}}$, maximal serum concentration; $C_{\min }$, minimum serum concentration; $\mathrm{CL} / \mathrm{F}$, apparent clearance; $\% \mathrm{CV}$, coefficient of variation expressed as a percentage; NA, not available; $R$, accumulation factor; $t_{1 / 2}$, terminal half-life; $T_{\max }$, observed time to achieve $C_{\max }$

a Median (range)

${ }^{\mathrm{b}}$ Accumulation factor $R=\mathrm{AUC}_{\text {tau }}$ peginterferon $\alpha-2 \mathrm{~b}$ at week $8 / \mathrm{AUC}_{\text {tau }}$ peginterferon $\alpha-2 \mathrm{~b}$ at week 1

c $n=14 ; \mathrm{CL} / \mathrm{F}$ cannot be reported for some patients because $t_{1 / 2}$ cannot be determined accurately

${ }^{\mathrm{d}} n=19$; no concentration data were available for one patient at week 12

\section{Noncompartmental analysis}

Arithmetic means and coefficients of variation (\%CV) of the estimated PK parameters at weeks 1, 8, and 12 from the noncompartmental PK analysis $(n=20)$ are summarized in Table 3. Peginterferon $\alpha-2 b$ was well-absorbed following SC administration, with a median $T_{\max }$ of $24 \mathrm{~h}$. Doserelated decreases in $C_{\max }, C_{\text {avg }}, C_{\min }$, and $\mathrm{AUC}_{\text {tau }}$ were observed following a dose decrease from 6 to $3 \mu \mathrm{g} /$ (kg week). After 8 weeks of dosing at $6 \mu \mathrm{g} /(\mathrm{kg}$ week) in the induction phase, the accumulation factor $(R)$ was 1.69 . $\mathrm{CL} / \mathrm{F}$ values demonstrated that the dosage change from induction to the maintenance phase did not appreciably affect estimates of CL/F. Mean half-life estimates ranged from 43 to $51 \mathrm{~h}$. PK parameters showed moderate interpatient variability with $\% \mathrm{CV}$ at weeks 1 and 8 ranging from 38 to $56 \%$; variability was slightly lower at week 12 (range 24-43\%; Table 3). In general, this regimen produced sustained exposure to peginterferon $\alpha-2 b$ at these dosages.

The arithmetic means of observed peginterferon $\alpha-2 b$ concentrations over time are shown in Fig. 1. Although the dose level changed from 6 to $3 \mu \mathrm{g} /(\mathrm{kg}$ week) at week 9 (maintenance phase), mean trough levels remained similar at weeks 10, 11, and 12. Mean predose (trough) serum concentrations $(\% \mathrm{CV})$ at weeks 10,11 , and 12 were 684 (30\%), $526(56 \%)$, and $626(43 \%) \mathrm{pg} / \mathrm{mL}$, respectively.

To estimate whether steady state had been reached by weeks 10 and 11, ratios of the least square (LS) mean trough concentrations were calculated. The LS means were 1.31 [90\% confidence interval (CI) 1.07-1.61] for week 10 versus the average of weeks 11 and 12 and 0.74 (90\% CI 0.58-0.93) for week 11 versus week 12. Conventionally, steady-state conditions are indicated by LS mean ratios between 0.80 and 1.25 [21]. Therefore, steady state could not be conclusively demonstrated.

At present, most patients diagnosed with stage III melanoma in the United States have disease in a single sentinel

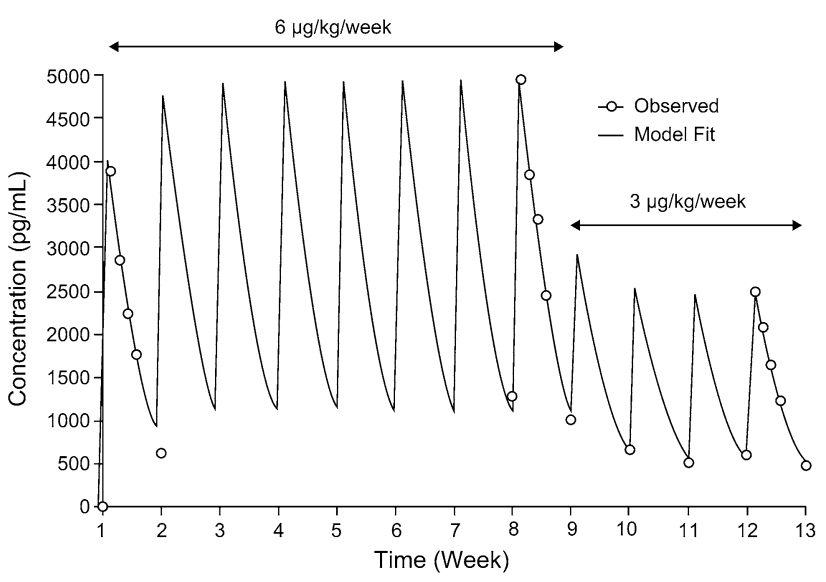

Fig. 1 Mean serum concentration-time profiles of peginterferon $\alpha-2 b$ following once-weekly subcutaneous dosing in patients who completed 12 weeks of protocol-specified therapy without dose modification and who had complete pharmacokinetic samples for weeks 1,8 , and $12(n=20)$. Experimentally observed values (open circles) as well as the curve-fitted line using a one-compartment pharmacokinetic model are included

node, which is referred to as $\mathrm{N} 1$ disease. In the EORTC 18991 trial, patients with N1 (N1a and N2a) disease appeared to derive benefit from adjuvant peginterferon $\alpha-2 b$ therapy $[15,22]$. Therefore, we also analyzed PK parameters for the subset of patients with N1a melanoma $(n=5)$. The results were consistent with those observed for all patients, although the exposure was marginally higher at week 8 (data not shown). As a result of the limited sample size for patients with $\mathrm{N} 1$ disease, no statistical analyses were conducted to compare the exposures in patients with different stages of disease.

\section{One-compartmental PK analyses}

The PK profiles for SC dosing can be described by a onecompartmental PK model with first-order absorption and 
first-order elimination. The one-compartmental model-predicted concentration-time profile is shown in Fig. 1. After the peak is reached, serum levels follow a monoexponential decay. The observed and model-predicted concentrations correlate well, as demonstrated in Fig. 1. The model describes the concentration-time profiles for the induction treatment of $6 \mu \mathrm{g} /(\mathrm{kg}$ week) from weeks $1-8$ as well as the initial portion of the maintenance phase at $3 \mu \mathrm{g} /(\mathrm{kg}$ week $)$ (serum trough levels at weeks 9-11 and a full profile at week 12). The consistent fit of this model across two dose levels suggests linear kinetics of peginterferon $\alpha-2 b$ through this dose range. Based on this model-predicted concentration-time profile, steady state was reached at week 4 following $6 \mu \mathrm{g} /(\mathrm{kg}$ week) SC dosing, and there was no apparent additional accumulation during the initial maintenance phase at $3 \mu \mathrm{g} /(\mathrm{kg}$ week) (weeks 9-12; Fig. 1). Therefore, this PK model provides a reasonable estimate of exposure to peginterferon $\alpha-2 b$ for use in the integrated PK/PD model.

\section{PK/PD modeling}

PK/PD models were developed in order to characterize the association between exposure and ANC or ALT response after SC administration of peginterferon $\alpha-2 b$ and to provide a model of the effect of dose reduction on these parameters.

\section{Effects of peginterferon $\alpha$ - $2 b$ on ANC}

Using pooled data from our patients with high-risk melanoma $(n=32)$ along with those from patients with advanced solid tumors $(n=34)$ [2], a nonlinear PK/PD relationship is seen between $\mathrm{AUC}_{\text {tau }}$ and change in $\mathrm{ANC}$ from baseline (Fig. 2). With increasing exposure to peginterferon $\alpha-2 b$, the ANC decreased relatively rapidly when $\mathrm{AUC}_{\mathrm{tau}}<200,000 \mathrm{pg} \mathrm{h} / \mathrm{mL}$, but the relationship between $\mathrm{AUC}_{\text {tau }}$ and change in ANC from baseline appeared to reach the maximum effect at exposure $\left(\mathrm{AUC}_{\text {tau }}\right)$ $400,000 \mathrm{pg} \mathrm{h} / \mathrm{mL}$, with $<10 \%$ decline of ANC between 200,000 and $600,000 \mathrm{pg} \mathrm{h} / \mathrm{mL}$.

The median ANC change (\% baseline) over time after weekly SC administration of peginterferon $\alpha-2 b$ demonstrated a rapid onset of effect within 1 week then a plateau between weeks 2 and 8 ; when the dose changed to $3 \mu \mathrm{g} /$ (kg week), a slow return towards baseline level followed (Fig. 3a). The low number of patients at week $6(n=4)$ and the interpatient variability account for the wide error bars at this time point. Particularly, one patient had an ANC increase of $38 \%$ from baseline due to ANC rebound following treatment cessation at weeks 4 and 5 [at week 6 ANC was measured before dosing was resumed at $6 \mu \mathrm{g} /(\mathrm{kg}$ week $)]$.

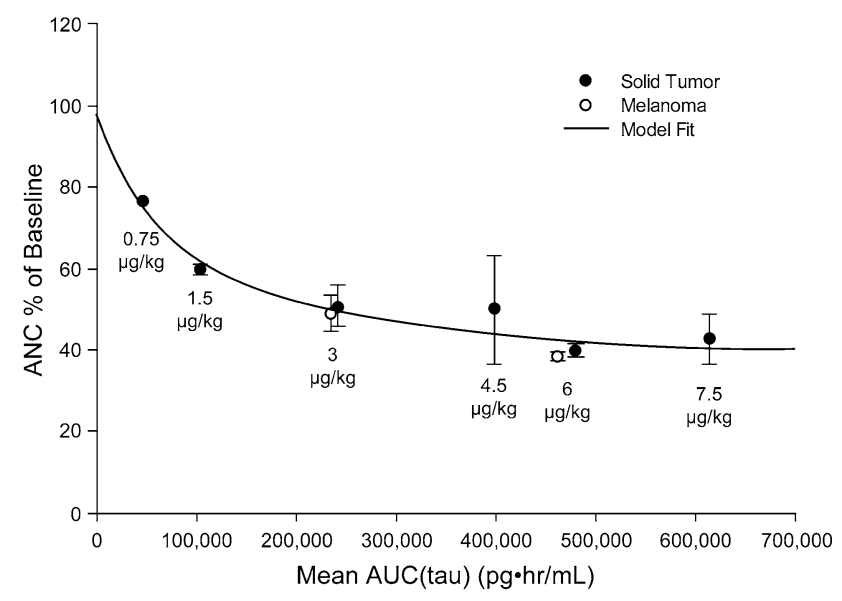

Fig. 2 Relationship between peginterferon $\alpha-2 b$ area under the concentration-time curve during the dosing interval $\left(\mathrm{AUC}_{\mathrm{tau}}\right)$ and absolute neutrophil count (ANC) changes as a percentage of baseline in patients with melanoma $(n=32)$ and solid tumors $(n=34)$ receiving various doses of peginterferon $\alpha-2 b$ (3-12 patients in each dose group)

The one-compartment PK model linked with an indirect response $\mathrm{PD}$ model appears to reasonably capture the time course of the ANC. We simulated the effects of a reduction in peginterferon $\alpha-2 b$ dose at weeks $9-12$ on ANC. Dose modification to 3,2 , or $1 \mu \mathrm{g} /(\mathrm{kg}$ week) resulted in an ANC increase of 13,22 , and $38 \%$, respectively. In the absence of dose reduction, i.e., peginterferon $\alpha-2 \mathrm{~b}$ dose kept at $6.0 \mu \mathrm{g} /$ (kg week), ANC levels will remain similar to those between weeks 2 and 8 (33\% of baseline value). The observed AUC increases during the maintenance phase (weeks 9-12) in this study were in line with model predictions (Fig. 3a). Similar results were observed when PK/PD modeling was carried out using data only from patients who received full doses and provided all blood samples in the PK phase. A more detailed description of the model, together with goodness-of-fit plots, is provided in the online resource material (supplementary methods, supplementary Table 1 and Fig. 1).

\section{Effects of peginterferon $\alpha-2 b$ on $A L T$}

The observed and predicted changes in median ALT with time are shown in Fig. 3b. The ALT profile demonstrates a delayed onset with a peak and trough pattern, with ALT levels gradually increasing over time and reaching a maximum between weeks 5 and 8 in the range of 2.5-3x baseline level. When the dose changed to $3 \mu \mathrm{g} /(\mathrm{kg}$ week) (week 9), ALT levels gradually decreased to approximately $2 \times$ baseline values between weeks 10 and 12.

As shown in Fig. 3b, the indirect stimulatory response model could describe the average trend of ALT change 

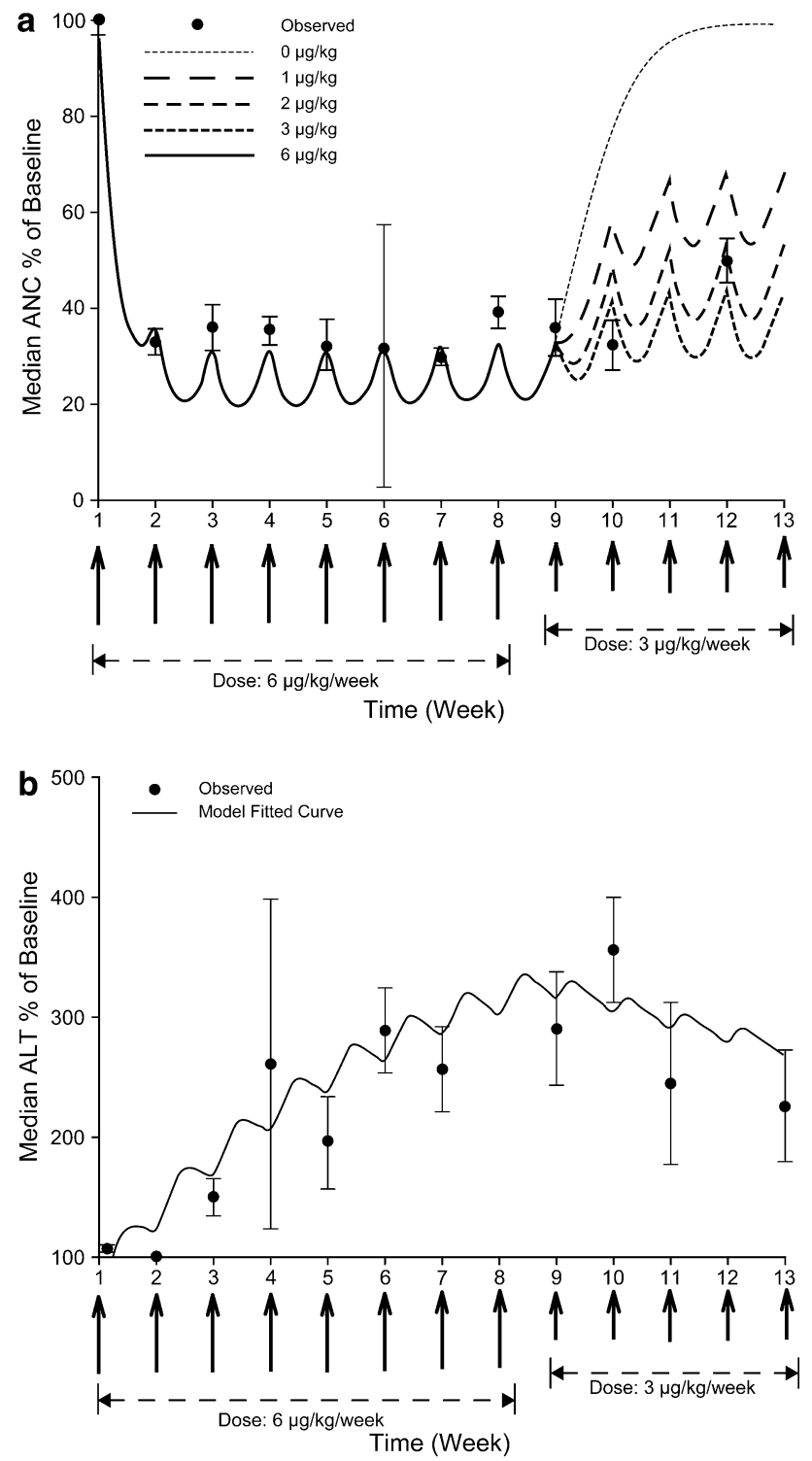

Fig. 3 Observed and predicted values of percentage changes from baseline in a median absolute neutrophil count (ANC) and b median alanine aminotransferase (ALT) levels. Simulation of percentage changes from baseline in median ANC/ALT with induction dose at $6 \mu \mathrm{g} /(\mathrm{kg}$ week) from weeks 1 to 8 , and dose modification of peginterferon $\alpha-2 b$ to $3,2,1$, or $0 \mu \mathrm{g} /(\mathrm{kg}$ week) during weeks $9-12$

following SC administration of peginterferon $\alpha-2 b$, but the fit was not as good as the ANC model. The individual ALT levels were highly variable; a notably large standard error was observed at week 4 . Therefore, this model should be treated with caution. Further details are provided in the online resource material (supplementary methods and supplementary Table 1). Similar results were observed when PK/PD modeling was carried out using data only from patients who received full doses and provided all blood samples in the PK phase.

\section{Discussion}

Peginterferon $\alpha-2 b$ is assuming a role in melanoma therapy based on the recent pivotal EORTC 18991 trial showing its efficacy in resected stage III melanoma [15]. The dose used in the EORTC 18991 trial, $6 \mu \mathrm{g} /(\mathrm{kg}$ week) for 8 weeks followed by $3 \mu \mathrm{g} /(\mathrm{kg}$ week) for up to 5 years, is much higher than that used for hepatitis C [1.5 $\mathrm{gg} /(\mathrm{kg}$ week $)]$. Prior to the current study being initiated, there were only limited data on systemic exposure to high-dose peginterferon $\alpha-2 b$, and no data were available for melanoma patients receiving adjuvant therapy.

We show that peginterferon $\alpha-2 b$ was well-absorbed following SC administration. Some accumulation occurred following 8 weeks of treatment at $6 \mu \mathrm{g} /(\mathrm{kg}$ week $)$. The $\mathrm{AUC}_{\text {tau }}$ for the $6 \mu \mathrm{g} /(\mathrm{kg}$ week) dose in this study was comparable with that observed in a small phase I/II trial [2] in patients with advanced solid tumors, although the $\mathrm{AUC}_{\text {tau }}$ for $3 \mu \mathrm{g} /(\mathrm{kg}$ week) was lower in our study [2]. As expected, the $\mathrm{CL} / \mathrm{F}$ of peginterferon $\alpha-2 \mathrm{~b}$ was greatly reduced compared with the clearance of native interferon $\alpha-2 b(231 \mathrm{~mL} /$ $\mathrm{h} \mathrm{kg}$ ) [23]. Therefore, peginterferon $\alpha-2 b$ has an approximately 10 -fold longer $t_{1 / 2}$ as compared with native interferon $\alpha-2 \mathrm{~b}$ (43-51 vs. $4 \mathrm{~h}$, respectively). The $\mathrm{CL} / \mathrm{F}$ and $t_{1 / 2}$ in patients with melanoma were similar to those reported in patients with hepatitis C (22 mL/h kg and $27-39 \mathrm{~h}$, respectively). The $C_{\max }$ observed here was $3,980-5,070 \mathrm{pg} / \mathrm{mL}$ at the $6 \mu \mathrm{g} /(\mathrm{kg}$ week) dose and $2,620 \mathrm{pg} / \mathrm{mL}$ at the $3 \mu \mathrm{g} /$ ( $\mathrm{kg}$ week) dose (measured at $24 \mathrm{~h}$ ). In a hepatitis C study, $C_{\max }$ varied between 15 and $44 \mathrm{~h}$ postdose [23]. Hence, the $C_{\max }$ in the current study could be potentially underestimated for some patients since we collected the first postdose PK sample at $24 \mathrm{~h}$. The $C_{\text {avg }}$ observed in the current study for the $3 \mu \mathrm{g} /(\mathrm{kg}$ week) dose $(1,400 \mathrm{pg} / \mathrm{mL})$ was comparable to the mean serum concentration observed in the limited PK analysis of the 18991 study $(1,434 \mathrm{pg} / \mathrm{mL})$. There is a difference in mean trough estimate at the $3 \mu \mathrm{g} /$ (kg week) dose level between the 18991 PK study $(1,069 \mathrm{pg} / \mathrm{mL})$ and the present study $(626 \mathrm{pg} / \mathrm{mL})$. This may be because the timing of sampling to assess trough levels in the 18991 PK study was not as rigorous as in our study, and the 18991 analysis may have included post-dose samples, potentially leading to an overestimation of mean trough concentration [16]. In addition, PK sampling took place over a much longer time period for the 18991 PK analysis compared with the present study (up to 5 years vs. 12 weeks, respectively).

The comparison of pegylated and native interferon $\alpha-2 b$ doses used in clinical trials is complicated by the differing measurement methods and units, schedules, and the specific activities. Data in patients with hepatitis $\mathrm{C}$ show that $0.25 \mu \mathrm{g} /(\mathrm{kg}$ week) peginterferon $\alpha$-2b results in a similar anti-viral effect as $9 \mathrm{MIU} /$ week (3 MIU three times weekly) native interferon $\alpha$-2b. Extrapolating from this 
data, $6.0 \mu \mathrm{g} /(\mathrm{kg}$ week) may achieve exposure comparable with that of interferon $\alpha-2 b$ at $20 \mathrm{MIU} / \mathrm{m}^{2} /$ day intravenous infusion five times weekly (180 MIU/week). The observed $\mathrm{AUC}_{\mathrm{tau}}$ in patients with melanoma confirmed the extrapolation. We observed that SC dosing of $6 \mu \mathrm{g} /(\mathrm{kg}$ week) for 8 weeks followed by $3 \mu \mathrm{g} /(\mathrm{kg}$ week) was well tolerated with toxicity similar to that observed in the EORTC 18991 trial; based on our experience with high-dose native interferon $\alpha-2 b$ in patients with melanoma, it appeared to us that our patients had lower incidence of fever and chills, but more skin rashes and hypertriglyceridemia. The data suggest that high-dose peginterferon $\alpha-2 b$ has a better overall tolerability profile compared with high-dose native interferon $\alpha$-2b. In phase I/II studies, peginterferon $\alpha$-2b has also been shown to be well tolerated and clinically active in chronic myelogenous leukemia and renal cell cancer [13].

We developed PK/PD models and performed simulations in order to visualize the effect of peginterferon $\alpha-2 b$ exposure on ANC and ALT levels, as these are clinically relevant interferon toxicities. We modeled the relationship between peginterferon $\alpha-2 \mathrm{~b} \quad \mathrm{AUC}_{\mathrm{tau}}$ and the observed reduction in ANC using data from our study as well as data from a prior study in patients with advanced solid tumors [2]. This analysis shows a steep dose response followed by a plateau around AUC $400,000 \mathrm{pg} \mathrm{h} / \mathrm{mL}$. The PK/PD model predicts that the ANC nadir is reached approximately 2 weeks after treatment initiation. The trough peginterferon $\alpha-2 \mathrm{~b}$ concentration $\left(C_{\min }\right)$ closely mirrors the ANC nadir (Fig. 3a). A similar relationship between peginterferon $\alpha-2 b$ dose and ANC changes after 4 weeks of dosing was reported in patients with hepatitis C [23]. Future studies with more intensive PD and PK sampling will assist in confirming that the wave-like fluctuation in ANC levels shown in Fig. 3a occur in clinical practice, as predicted by the PK/PD model.

Since clinicians may be interested in peginterferon $\alpha-2 b$ dose reductions for excessive neutropenia, we also simulated the effect of dose reduction on the ANC. If peginterferon $\alpha-2 b$ dose is reduced from $6 \mu \mathrm{g} /(\mathrm{kg}$ week) to 0 , the ANC trends upward rapidly. While stepwise dose reductions $(3-2-1 \mu \mathrm{g} / \mathrm{kg}$ ) were prescribed in the EORTC 18991 trial, the Imax model used here suggests that treatment should be interrupted and resumed at a much lower dose (50 or $33 \%$ of starting dose) upon resolution of neutropenia, if grade 3 or 4 neutropenia is observed. Clearly, future trials should examine this clinically relevant issue.

Progressive transaminase elevations (reversible upon discontinuation of the drug) are a major factor in the ability of patients with melanoma to tolerate the full prescribed dose of peginterferon $\alpha-2 b$; ALT elevation is an important PD surrogate of high-dose interferon effect on the liver [3$5,15]$. Although the number of patients with grade 3 ALT elevations in the current study is low (four patients; 13\%), the frequency is consistent with the EORTC 18991 trial data (10\% grade 3 liver function test AEs) [15]. There were no grade 4 ALT elevations in our study. We explored this relationship using a fully integrated PK/PD model. Our model predictions for ALT did not appear to fit our observed data as well as for ANC, so further evaluation of this aspect of peginterferon administration is also clearly warranted.

In this study, the PK profile of high-dose peginterferon $\alpha-2 b$ in patients with resected high-risk melanoma is described. The tolerability and safety of this dose and schedule appears to be broadly similar to that seen in previous studies. The PK/PD model of the effects of peginterferon $\alpha-2 b$ exposure on ANC provides a useful framework. In contrast to high-dose interferon $\alpha$-2b, hematologic and/or hepatic toxicity was less common and primarily grade 1 or 2 in severity with peginterferon $\alpha-2 b \quad 6 \mu \mathrm{g} /(\mathrm{kg}$ week $)$ (induction) and $3 \mu \mathrm{g} /(\mathrm{kg}$ week) (maintenance). Based on the safety profile and PK data, combined with greater dosing convenience, peginterferon $\alpha-2 b$ may potentially improve compliance and efficacy of adjuvant therapy for patients with high-risk melanoma.

Acknowledgments Our profound thanks go to all of the patients and their families who participated in this trial. We would like to thank Emilio Batageli, MD (Unidad de Farmacologia Clinic de Aclires Argentina SRL Hospital Privado Modelo, Buenos Aires, Argentina), Alexander Guminski, MD, PhD and Warren Joubert, MD (Princess Alexandra Hospital, Woolloongabba, Queensland, Australia), and Sabe Sabesan, MD (Mater Misericordiae Hospital, Townsville, Queensland, Australia) for their contributions. The authors were involved in all aspects of developing, writing, and reviewing the manuscript and also acknowledge the medical writing assistance of Meenakshi Subramanian, PhD and Helen Varley, PhD at Evidence Scientific Solutions, which was supported by Schering-Plough Corporation (now Schering Corp., a Division of Merck \& Co.). We particularly acknowledge the staff of the Clinical Research Unit at the Moffitt Cancer Center, where the majority of patients on this study were treated.

Conflict of interest statement A.I.D. has received research funding or funding for equipment or drugs from Schering-Plough (now Schering Corp., a Division of Merck \& Co.). C.X. is a full-time employee of Merck Research Laboratories (formerly Schering-Plough Research Institute). At the time of analysis and writing, A.Y. was a full-time employee of Schering-Plough Research Institute and is a stock owner for Merck \& Co. W.J.H. has received research funding and drugs from, and is consultant to, Schering-Plough (now Schering Corp., a Division of Merck \& Co.). V.K.S. is a paid consultant to and on the speakers' bureau of Schering-Plough (now Schering Corp., a Division of Merck \& Co.). P.U., S.A., N.E.P., L.C.F., and R.D.C. declared no conflicts of interest.

Open Access This article is distributed under the terms of the Creative Commons Attribution Noncommercial License which permits any noncommercial use, distribution, and reproduction in any medium, provided the original author(s) and source are credited.

\section{References}

1. Foster GR (2004) Review article: pegylated interferons: chemical and clinical differences. Aliment Pharmacol Ther 20:825-830 
2. Bukowski R, Ernstoff MS, Gore ME et al (2002) Pegylated interferon alfa- $2 b$ treatment for patients with solid tumors: a phase I/II study. J Clin Oncol 20:3841-3849

3. Kirkwood JM, Strawderman MH, Ernstoff MS, Smith TJ, Borden EC, Blum RH (1996) Interferon alfa-2b adjuvant therapy of highrisk resected cutaneous melanoma: the Eastern Cooperative Oncology Group Trial EST 1684. J Clin Oncol 14:7-17

4. Kirkwood JM, Ibrahim JG, Sondak VK et al (2000) High- and lowdose interferon alfa- $2 b$ in high-risk melanoma: first analysis of intergroup trial E1690/S9111/C9190. J Clin Oncol 18:2444-2458

5. Kirkwood JM, Ibrahim JG, Sosman JA et al (2001) High-dose interferon alfa- $2 \mathrm{~b}$ significantly prolongs relapse-free and overall survival compared with the GM2-KLH/QS-21 vaccine in patients with resected stage IIB-III melanoma: results of intergroup trial E1694/S9512/C509801. J Clin Oncol 19:2370-2380

6. Verma S, Quirt I, McCready D, Bak K, Charette M, Iscoe N (2006) Systematic review of systemic adjuvant therapy for patients at high risk for recurrent melanoma. Cancer 106:1431-1442

7. Wheatley K, Ives N, Eggermont A et al (2007) Interferon- $\alpha$ as adjuvant therapy for melanoma: an individual patient data meta-analysis of randomised trials. J Clin Oncol (Meeting Abstracts) 25:8526

8. Kirkwood JM, Bender C, Agarwala S et al (2002) Mechanisms and management of toxicities associated with high-dose interferon alfa-2b therapy. J Clin Oncol 20:3703-3718

9. Carrat F, Bani-Sadr F, Pol S et al (2004) Pegylated interferon alfa$2 \mathrm{~b}$ vs standard interferon alfa- $2 \mathrm{~b}$, plus ribavirin, for chronic hepatitis C in HIV-infected patients: a randomized controlled trial. JAMA 292:2839-2848

10. Khalili M, Bernstein D, Lentz E, Barylski C, Hoffman-Terry M (2005) Pegylated interferon $\alpha$-2a with or without ribavirin in HCV/ HIV coinfection: partially blinded, randomized multicenter trial. Dig Dis Sci 50:1148-1155

11. Lindsay KL, Trepo C, Heintges T et al (2001) A randomized, double-blind trial comparing pegylated interferon alfa- $2 \mathrm{~b}$ to interferon alfa-2b as initial treatment for chronic hepatitis C. Hepatology 34:395-403

12. Zeuzem S, Feinman SV, Rasenack J et al (2000) Peginterferon alfa-2a in patients with chronic hepatitis C. N Engl J Med 343:1666-1672

13. Bukowski RM, Tendler C, Cutler D, Rose E, Laughlin MM, Statkevich P (2002) Treating cancer with PEG Intron: pharmaco- kinetic profile and dosing guidelines for an improved interferonalpha-2b formulation. Cancer 95:389-396

14. Tagliaferri P, Caraglia M, Budillon A et al (2005) New pharmacokinetic and pharmacodynamic tools for interferon-alpha (IFN- $\alpha$ ) treatment of human cancer. Cancer Immunol Immunother 54:1-10

15. Eggermont AM, Suciu S, Santinami M et al (2008) Adjuvant therapy with pegylated interferon alfa- $2 \mathrm{~b}$ versus observation alone in resected stage III melanoma: final results of EORTC 18991, a randomised phase III trial. Lancet 372:117-126

16. Eggermont AM, Bouwhuis MG, Kruit WH et al (2009) Serum concentrations of pegylated interferon alpha- $2 b$ in patients with resected stage III melanoma receiving adjuvant pegylated interferon alpha-2b in a randomized phase III trial (EORTC 18991). Cancer Chemother Pharmacol 65:671-677

17. Ong JP, Younossi ZM (2004) Managing the hematologic side effects of antiviral therapy for chronic hepatitis C: anemia, neutropenia, and thrombocytopenia. Cleve Clin J Med 71(Suppl 3):S17S21

18. Gibaldi M, Perrier D (1982) Pharmacokinetics, 2nd edn. Marcel Dekker, Inc, New York

19. Mager DE, Jusko WJ (2002) Receptor-mediated pharmacokinetic/ pharmacodynamic model of interferon- $\beta$ 1a in humans. Pharm Res 19:1537-1543

20. Nieforth KA, Nadeau R, Patel IH, Mould D (1996) Use of an indirect pharmacodynamic stimulation model of MX protein induction to compare in vivo activity of interferon alfa-2a and a polyethylene glycol-modified derivative in healthy subjects. Clin Pharmacol Ther 59:636-646

21. U.S. Department of Health \& Human Services, Food and Drug Administration \& Center for Drug Evaluation and Research (CDER). Guidance for Industry: Statistical Approaches to Establishing Bioequivalence. January 2001. http://www.fda.gov/ downloads/Drugs/GuidanceComplianceRegulatoryInformation/ Guidances/ucm070244.pdf. Accessed 5 November 2009

22. Sondak VK, Flaherty LE (2008) Adjuvant therapy of melanoma: is pegylated interferon alfa- $2 \mathrm{~b}$ what we've been waiting for? Lancet 372:89-90

23. Glue P, Fang JW, Rouzier-Panis R et al (2000) Pegylated interferon- $\alpha 2 \mathrm{~b}$ : pharmacokinetics, pharmacodynamics, safety, and preliminary efficacy data. Hepatitis C Intervention Therapy Group. Clin Pharmacol Ther 68:556-567 